\title{
Tabak-, Alkohol- und Cannabiskonsum bei jüngeren Jugendlichen in NRW
}

\author{
A. Leppin \\ M. Richter \\ K. Hurrelmann
}

Tabacco, Alcohol and Cannabis Use of Young Adolescents in NRW

\section{Zusammenfassung}

Während ein experimenteller Gebrauch psychoaktiver Substanzen im Jugendalter als normativ gelten und bis zu einem gewissen Grad auch dazu beitragen kann, Entwicklungsaufgaben zu bewältigen, ist ein früher regelmäßiger oder exzessiver Konsum problematisch. Im Rahmen der WHO-Studie „Health Behaviour in School-aged Children“ wurden im Jahr 2001/2002 3326 Schüler und Schülerinnen in NRW im Alter zwischen elf und 15 Jahren bezüglich ihrer Konsummuster von Tabak, Alkohol und Cannabis befragt. Etwa die Hälfte aller Befragten hat bereits mit Zigaretten experimentiert, $50 \%$ haben in der letzten Zeit Alkohol getrunken, $25 \%$ erste Erfahrungen mit Cannabis gemacht. Zu den regelmäßigen Rauchern und Alkoholkonsumenten gehören jedoch nur jeweils circa $15 \%$ der Schüler und Schülerinnen. Im internationalen Vergleich liegen die Jugendlichen aus NRW im Bezug auf Rauchen und Alkoholkonsum dennoch deutlich über dem Durchschnitt der anderen Länder auf einem vorderen Rangplatz.

\section{Schliuisselwörter}

Alkohol · Tabak · Cannabis · Jugendliche

\section{Abstract}

While experimental use of psychoactive substances during adolescence can be viewed as normative and to a certain extent adaptive in terms of coping with developmental tasks, an early habitual and/or excessive consumption pattern is problematic. Within the context of the WHO study "Health Behaviour in School-aged Children" in 2001/2002 332611 -15 year old students from North Rhine -Westphalia filled in a standardized questionnaire asking for their consumption patterns concerning tobacco, alcohol and cannabis. Almost half of the sample has experimented with cigarettes, $50 \%$ say they have recently drunk alcohol and $25 \%$ report first experiences with cannabis. In comparison the subgroup of those who smoke or drink alcohol regularly is - with a percentage of $15 \%$ - considerably smaller. However, comparing North Rhine-Westphalia to other countries from the HBSC study shows that in terms of smoking and drinking alcohol adolescents from North Rhine-Westphalia rank well above average.

Key words

Alcohol $\cdot$ tabacco $\cdot$ cannabis $\cdot$ adolescents
In der Adoleszenz erarbeiten sich Jugendliche zunehmend Autonomie und Unabhängigkeit von elterlichen Einflüssen und experimentieren in diesem Zusammenhang mit einer Fülle für sie neuer Verhaltensweisen, die das Erwachsenenalter vorwegnehmen sollen. Eine besondere Rolle spielt hierbei der Konsum legaler und illegaler Drogen. Ihre Funktionalität beziehen die psychoaktiven Substanzen anfänglich vor allem aus sozialen
Motiven. Ziel des Verhaltens ist also nicht notwendigerweise der Genuss beim Konsum, sondern die Alltagsdrogen dienen auch als Mittel der Bewältigung von Entwicklungsaufgaben wie dem Aufbau von Beziehungen zu gleichaltrigen Peers, der Lebensstilentwicklung, der Abgrenzung von den Eltern und der Identitätsbildung [1-4]. Erst mit der Habituierung des Verhaltens, die bei bestimmten Substanzen wie Nikotin allerdings rela-

Institutsangaben

Fakultät für Gesundheitswissenschaften, Universität Bielefeld

Korrespondenzadresse

PD Dr. Anja Leppin · Fakultät für Gesundheitswissenschaften, Universität Bielefeld · PF 100131 .

33501 Bielefeld · E-mail: Anja.Leppin@uni-bielefeld.de

Bibliografie

Suchttherapie 2005; 6: 4-10 @ Georg Thieme Verlag KG Stuttgart · New York

DOI 10.1055/s-2005-858058

ISSN 1439-9903 
tiv rasch eintreten kann [5], löst sich der Konsum aus diesem funktionalen Zusammenhang und wird dann (auch) zum Selbstzweck. In diesem Sinn ist vor allem der experimentelle und gemäßigte Konsum von Alltagsdrogen quasi-normativer Bestandteil des Erwachsenwerdens. Abstinenzforderungen - dies gilt besonders für die in der Erwachsenenwelt allseits akzeptierte Alltagsdroge Alkohol - sind deshalb zumeist unrealistisch und auch nicht per se mit guter psychosozialer Anpassung gleichzusetzen [6, 7].

Andererseits darf nicht übersehen werden, dass ein sehr früher wie auch ein häufiger oder gar exzessiver Substanzkonsum bei Jugendlichen als Risikofaktor für Entwicklungsstörungen gilt und gesundheitliche und soziale Probleme wie Abhängigkeit, Schulschwierigkeiten, aggressives Verhalten, Intoxikationen, Unfälle etc. zur Folge haben kann. Oft sind solche prekären Konsumformen Ausdruck fehlgeleiteter Strategien, mit situativen Anforderungen umzugehen, die die eigenen Handlungskompetenzen übersteigen. Häufig ist dies dann der Fall, wenn normative Entwicklungsaufgaben durch zusätzliche akute oder überdauernde Belastungssituationen im Lebensalltag, wie z. B. Konflikte mit den Eltern, Anerkennungsprobleme in der Gleichaltrigengruppe, schulische Leistungsschwierigkeiten etc., verstärkt werden und die betroffenen Jugendlichen gleichzeitig nur ein geringes Maß psychosozialer Ressourcen besitzen [8-11]. Selbst Problemkonsum bleibt allerdings häufig jugendphasenspezifisch [12,13], wobei es vor allem dann zu einer Verstetigung zu kommen scheint, wenn bereits in der frühen Kindheit bestimmte Problemverhaltensweisen wie Aggressivität, Hyperaktivität etc. aufgetreten sind [13-15]. Während der experimentelle Gebrauch psychoaktiver Substanzen bei Jugendlichen also nicht per se als kritisch gesehen werden muss, ist ein früher und dabei besonders ein regelmäßiger Konsum als Risikofaktor für die weitere gesundheitliche und psychosoziale Entwicklung zu betrachten. Im folgenden Beitrag sollen empirische Befunde zu aktuellen Konsummustern und dabei besonders zum regelmäßigen Konsum von Tabak, Alkohol und Cannabis bei 11- bis 15-jährigen Jugendlichen aus NRW vorgestellt und mit Daten aus anderen Bundesländern und anderen Nationen verglichen werden.

\section{Methode}

Datenbasis ist die deutsche und nordrhein-westfälische Stichprobe der internationalen Studie „Health Behaviour in Schoolaged Children (HBSC)“ aus dem Jahr 2002. Ziel der alle vier Jahre durchgeführten Studie ist es, Daten über die Gesundheit und das Gesundheitsverhalten von Jugendlichen im Alter von 11, 13 und 15 Jahren zu sammeln und umfassende Veränderungen dieses Verhaltens im Lauf der Zeit zu bestimmen [16, 17]. An der aktuellen Studie beteiligten sich Forschergruppen aus insgesamt 35 Ländern in Europa und Nordamerika. Die Durchführung des nationalen Surveys obliegt dabei den Forschungsteams aus den einzelnen Mitgliedsländern nach festgelegten Richtlinien für die Stichprobenauswahl, die methodische Durchführung und die Aufbereitung der Daten, die in einem internationalen Untersuchungsprotokoll festgehalten werden. Als Erhebungsinstrument dient ein standardisierter Fragebogen zum Selbstausfüllen.
Die Stichprobe wurde in allen teilnehmenden Länder durch ein Clusterverfahren auf Basis von Schulklassen oder in Ausnahmefällen von Schulen als primäre Stichprobeneinheiten gezogen, so dass die Altersgruppen der 11-, 13- und 15-Jährigen mit einer Abweichung von etwa einem halben Jahr repräsentiert sind. Fast alle Länder sind mit einer repräsentativen Bevölkerungsstichprobe an der Studie beteiligt. Der deutsche Survey bildet eine der Ausnahmen, insofern er auf regionalen Stichproben aus den vier Bundesländern Nordrhein-Westfalen, Hessen, Berlin und Sachsen basiert [für eine ausführliche Stichprobenbeschreibung siehe 18,19$]$. Diese strukturtypische Stichprobe für Deutschland besteht aus 5650 Kindern und Jugendlichen zwischen 11 und 15 Jahren. In Tab. 1 ist die Verteilung der Stichprobe für die einzelnen Bundesländer dargestellt. Mit Ausnahme der Beschreibung der innerdeutschen Vergleiche werden im Folgenden ausschließlich Daten der nordrhein-westfälischen Stichprobe $(\mathrm{n}=3339)$ dargestellt.

Tab. 1 Strukturtypischer Datensatz für Deutschland nach Bundesland, Alter und Geschlecht

\begin{tabular}{llll}
\hline Bundesland & $\begin{array}{l}\text { gesamt } \\
\boldsymbol{n}\end{array}$ & $\begin{array}{l}\text { Jungen } \\
\%(\boldsymbol{n})\end{array}$ & $\begin{array}{l}\text { Mädchen } \\
\%(\boldsymbol{n})\end{array}$ \\
\hline Nordrhein-Westfalen & 3339 & $58,4(1626)$ & $59,8(1713)$ \\
\hline Hessen & 1081 & $19,3(539)$ & $18,9(542)$ \\
\hline Sachsen & 681 & $12,5(347)$ & $11,7(334)$ \\
\hline Berlin & 549 & $9,8(274)$ & $9,6(275)$ \\
\hline Alter & & & \\
$\quad$ 11-Jährige (ø 11,56) & 2100 & $50,5(1061)$ & $49,5(1039)$ \\
13-Jährige (ø 13,63) & 1801 & $48,9(880)$ & $51,1(921)$ \\
$\quad$ 15-Jährige (ø 15,65) & 1749 & $48,3(845)$ & $51,7(904)$ \\
\hline gesamt & 5650 & $49,3(2786)$ & $50,7(2864)$ \\
\hline
\end{tabular}

Der Tabakkonsum wurde in der HBSC-Studie mit Hilfe von zwei Fragen erhoben. Zum einen wurden die Kinder und Jugendlichen gefragt, ob sie schon einmal geraucht haben (Antwortkategorien: ja/nein). Zum anderen wurden sie gefragt, wie oft sie zurzeit rauchen. Die Jugendlichen konnten dabei angeben, ob sie ,jeden Tag“, ,mindestens einmal in der Woche, aber nicht täglich“, ,weniger als einmal in der Woche“ oder "nicht rauchen“. In Anlehnung an die Definition der Bundeszentrale für gesundheitliche Aufklärung [20] wurden Jugendliche, die mindestens einmal in der Woche oder täglich rauchen, als regelmäßige Raucher eingestuft. Ein Tabakkonsum von „weniger als einmal in der Woche“ gilt als gelegentlicher Konsum.

Der aktuelle Alkoholkonsum bzw. die aktuelle Konsumhäufigkeit wurde durch die Frage erfasst: „Wie oft hast du in letzter Zeit alkoholische Getränke (auch in kleinen Mengen) getrunken?", wobei zwischen vier Alkoholsorten 1. Bier, 2. Wein oder Sekt, 3. Schnaps oder Likör und 4. Mixgetränke oder Cocktails unterschieden wurde. Die Frage war mittels einer 5-stufigen Antwortskala mit den folgenden Ausprägungen zu beantworten: „jeden Tag“, ,jede Woche“, ,jeden Monat“, „seltener als einmal pro Monat" und „nie“. Aus den verschiedenen Angaben zum Alkoholkonsum wurde ein Gesamtindex gebildet, der jeweils 
die höchste Konsumhäufigkeit der einzelnen Getränkesorten berücksichtigt. Analog zum Tabakkonsum wurden die Angaben zu täglichem und mindestens einmaligem wöchentlichen Konsum zu einer Kategorie „regelmäßiger Konsum“ zusammengefasst. Um die Häufigkeit exzessiven Alkoholkonsums zu erheben, sollten die Jugendlichen angeben, wie häufig sie bereits soviel Alkohol getrunken haben, dass sie richtig betrunken waren (Antwortmöglichkeiten: „nie“, „einmal“, 2- bis 3-mal“, 4- bis 10-mal“ und „öfter als 10-mal). Der Konsum von Cannabis wurde ausschließlich bei 15-Jährigen erhoben. Die Schülerinnen und Schüler wurden dabei gefragt, ob sie (1.) jemals in ihrem bisherigen Leben und ob sie (2.) in den letzten zwölf Monaten Cannabis, Haschisch oder Marihuana genommen haben (Antwortvorgaben: „nie“, „ein- oder zweimal“, „3- bis 5-mal“, „6- bis 9-mal“, „10- bis 19-mal“, „20- bis 39-mal“, „40-mal und mehr“). Für die deskriptive Darstellung wurden Kreuztabellen verwendet. Geschlechtsund Altersunterschiede wurden bei nominalen oder kategorialen Variablen mit dem Chi-Quadrat-Test auf Unabhängigkeit der beiden Variablen überprüft. Darüber hinaus sind Odds Ratios (OR) und Konfidenzintervalle $(\mathrm{CI})$ angegeben, um das „relative Risiko“ für verschiedene Subgruppen besser abschätzen zu können.

\section{Ergebnisse}

\section{Häufigkeit des Tabakkonsums}

Fast die Hälfte aller 11- bis 15-Jährigen in NRW, nämlich 46,1\%, berichten 2001/2002, dass sie schon einmal geraucht haben. Erwartungsgemäß gibt es dabei signifikante Unterschiede in Abhängigkeit vom Alter bzw. der Klassenstufe $\left(\chi^{2}=640,08 ; d f=2\right.$; $\mathrm{p}<, 001$ ). Während die Lebenszeitprävalenz bei den 11-Jährigen mit 18,9\% noch eher gering ist, steigt sie innerhalb von zwei Jahren auf über $54 \%$ an, um dann in der 9 . Klassenstufe $70,5 \%$ zu erreichen, so dass bei den 15 -Jährigen nur noch knapp $30 \%$ ohne jede Raucherfahrung sind. Schlüsselt man die Lebenszeitprävalenz zusätzlich nach Alter und Geschlecht auf, wird deutlich, dass sich Jungen und Mädchen bezüglich ihres Rauchverhaltens in Klassenstufe 5 noch deutlich und signifikant unterscheiden: $24,4 \%$ der Jungen, jedoch nur 13,5\% der Mädchen geben an, bereits Zigaretten probiert zu haben $(\mathrm{OR}=2,07 ; \mathrm{CI}=1,54-2,77)$. Schon zwei Jahre später haben die Mädchen jedoch aufgeholt und die Prävalenzen überschreiten in beiden Gruppen die 50\%-Marke (54,6\% Jungen vs. 53,3\% Mädchen). Bis zur Klassenstufe 9 haben Mädchen mit einer Quote von 71,6 die Jungen mit $69,2 \%$ überholt; allerdings ist dieser Unterschied nicht signifikant.

Natürlich enthält die Lebenszeitprävalenz einen recht hohen Anteil an Experimentier- oder Probierkonsumenten, das heißt, der Anteil derjenigen, die häufiger konsumieren und deshalb als besonders anfällig für eine Habituierung zum abhängigen Raucher gelten müssen, ist deutlich niedriger zu veranschlagen. Daher bieten die Antworten auf die Frage nach dem aktuellen Tabakkonsum eher Aufschluss über den Anteil gefährdeter Jugendlicher (siehe Tab.2). Die große Mehrheit, nämlich 79,2\% gibt an, momentan nicht zu rauchen, $5,7 \%$ berichteten nur sporadischen Tabakkonsum, von weniger als einmal in der Woche. 15,1\% lassen sich dagegen als regelmäßige Konsumenten einstufen, wobei 11,6\% ihren eigenen Angaben zu Folge tägliche Raucher sind.
Auch für diesen regelmäßigen Konsum sind erhebliche Unterschiede zwischen den Klassen- bzw. Altersstufen zu verzeichnen (siehe Tab. 2). Rauchen bei den 11-Jährigen erst 2,3\% regelmäßig, steigt dieser Anteil auf fast ein Drittel aller Jugendlichen bei den 15-Jährigen. Dabei zeigen die Angaben für die drei Altersgruppen deutlich, wie aus gelegentlichem gewohnheitsmäßiger Konsum wird. Ist im Alter von elf Jahren der Anteil der gelegentlichen Raucher noch größer als der der regelmäßigen Konsumenten, hat sich dieses Verhältnis bereits mit 13 Jahren zu Gunsten der Gewohnheitskonsumenten verschoben, die dann bei den 15-Jährigen endgültig dominieren. Gelegentliche Raucher sind eher selten. Entweder man raucht gar nicht oder regelmäßig, wobei unter den 13- und 15-jährigen Gewohnheitsrauchern wiederum die täglichen Raucher deutlich häufiger vertreten sind als diejenigen, die nicht unbedingt jeden Tag rauchen. Während insgesamt keine Geschlechtsunterschiede auftreten, ist der Entwicklungsverlauf für Jungen und Mädchen durchaus unterschiedlich (Tab. 2). In Klassenstufe 5 fallen Jungen noch dreimal so häufig in die Kategorie der regelmäßigen Raucher wie Mädchen $(\mathrm{OR}=3,26 ; \mathrm{CI}=1,38-7,69)$. Bereits in Stufe 7 hat sich dieses Verhältnis jedoch angeglichen, und in Klasse 9 liegen die Mädchen, was den täglichen Konsum angeht, sogar leicht vorn, wobei dieser Unterschied jedoch nicht signifikant ist.

\section{Innerdeutscher Vergleich der Konsumquoten}

Im Vergleich der regelmäßigen Raucher und Raucherinnen zwischen den vier an der HBSC-Studie beteiligten Bundesländern NRW, Hessen, Sachsen und Berlin, liegen die nordrhein-westfälischen Jugendlichen im Jahr 2001/2002 mit 15,1\% auf dem zweiten Platz, wobei es zu Hessen (14,1\%) und Sachsen (16,1\%) allerdings keine signifikanten Unterschiede gibt. Deutlich ist dagegen die Differenz zu den mit einer Quote von 19,6\% führenden Berlinern $(\mathrm{OR}=.73 ; \mathrm{Cl}=.58-.93)$. Geschlechtsspezifische Analysen zeigen, dass diese Unterschiede auf die Subgruppe der Mädchen zurückgehen (NRW = 15,1\% vs. Berlin: 20,7\% OR = .68; CI = .49-.94), während für die Jungen keine bedeutsamen regionalen Unterschiede auftreten.

\section{Internationaler Vergleich der Konsumquoten}

Während die nordrhein-westfälischen Jugendlichen im innerdeutschen Vergleich also nicht aus dem Rahmen fallen, sieht dies im internationalen Kontext deutlich anders aus. So nimmt sowohl die Gesamtgruppe der deutschen Jugendlichen als auch die NRW-spezifische Stichprobe im Jahr 2001/2002 in punkto Rauchen einen Spitzenplatz ein [17, 21]. Die internationale Auswertung der HBSC-Studie unter Beteiligung von 35 Ländern zeigt, dass die nordrhein-westfälischen Jugendlichen den dritten Platz in den Rauchfrequenzraten bei den 15-Jährigen belegen und damit nur noch von Grönland und der Ukraine übertroffen werden. Bei den 13-Jährigen liegen die nordrhein-westfälischen Jugendlichen sogar auf dem zweiten Platz. Lediglich die 11-Jährigen Nordrhein-Westfalens rangieren im internationalen Vergleich zumindest nicht ganz oben, sondern nehmen Platz 7 ein. Allerdings bedeutet auch dieser siebte Rang bei den 11-Jährigen immer noch eine Platzierung im oberen Viertel der Verteilung. Insgesamt liegen die Schüler und Schülerinnen aus NRW damit deutlich über dem Durchschnitt aller beteiligten Nationen und Regionen. 
Tab. 2 Konsum psychoaktiver Substanzen und alkoholbedingte Rauscherfahrungen nach Alter und Geschlecht (Angaben in Prozent)

\begin{tabular}{|c|c|c|c|c|c|c|c|c|c|c|}
\hline & \multicolumn{2}{|l|}{ gesamt } & \multicolumn{2}{|l|}{ 11-Jährige } & \multicolumn{2}{|l|}{ 13-Jährige } & \multicolumn{2}{|l|}{ 15-Jährige } & \multirow{2}{*}{$\begin{array}{l}\text { Geschlechts- } \\
\text { effekt }{ }^{1} \\
\chi^{2}, d f, p\end{array}$} & \multirow{2}{*}{$\begin{array}{l}\text { Alterseffekt }^{2} \\
\chi^{2}, d f, p\end{array}$} \\
\hline & $\hat{0}$ & q & $\hat{\jmath}$ & q & $\hat{0}$ & q & $\hat{\jmath}$ & q & & \\
\hline Nichtraucher & 79,1 & 79,2 & 92,2 & 96,6 & 78,0 & 78,3 & 63,6 & 60,0 & & \\
\hline Gelegenheitsraucher & 5,7 & 5,7 & 4,2 & 2,2 & 8,3 & 7,5 & 4,8 & 8,0 & ns & $\begin{array}{l}\chi^{2}=435,4 \\
d f=6, p<0,001\end{array}$ \\
\hline Alkoholkonsum & $(n=1597)$ & $(n=1671)$ & $(n=603)$ & $(n=603)$ & $(n=510)$ & $(n=527)$ & $(n=477)$ & $(n=536)$ & & \\
\hline kein Alkohol & 49,2 & 53,5 & 72,3 & 85,7 & 50,6 & 51,8 & 18,7 & 19,4 & & \\
\hline gelegentlicher Konsum & 33,6 & 34,9 & 23,2 & 12,9 & 38,4 & 39,1 & 41,1 & 55,4 & $\begin{array}{l}\chi^{2}=23,64 \\
d f=4, p<0,001\end{array}$ & $\begin{array}{l}\chi^{2}=1069,37 \\
d f=8, p<0,001\end{array}$ \\
\hline regelmäßiger Konsum & 17,3 & 11,6 & 4,5 & 1,3 & 10,9 & 9,1 & 40,3 & 25,2 & & \\
\hline zweimal oder mehr & 18,0 & 14,9 & 4,0 & 0,8 & 11,2 & 10,6 & 43,1 & 35,4 & & \\
\hline $\begin{array}{l}\text { Cannabiskonsum } \\
\text { (in den letzten zwölf } \\
\text { Monaten) }\end{array}$ & & & & & & & $(n=464)$ & $(n=526)$ & & \\
\hline nie & & & - & - & - & - & 77,6 & 86,5 & $\begin{array}{l}\chi^{2}=22,3 \\
d f=6, p<0,001\end{array}$ & - \\
\hline mindestens einmal & & & - & - & - & - & 22,4 & 13,5 & & \\
\hline
\end{tabular}

${ }^{1}$ Geschlechtseffekt getestet über alle Altersgruppen (Spalte „gesamt“)

2 Alterseffekt getestet für Jungen und Mädchen gemeinsam; Quelle: HBSC-Surveys Nordrhein-Westfalen

\section{Entwicklung des Rauchverhaltens}

Ein Blick auf die Entwicklung des Rauchverhaltens seit Beginn der 90er-Jahre lässt günstigstenfalls einen verhaltenen Optimismus bezüglich möglicher Reduktionen der Raucherquoten zu. Nach einem deutlichen Anstieg, der zwischen 1993/94 und 1997/98 für fast alle Jugendlichen bis 15 Jahren - mit Ausnahme der jüngeren Mädchen, die von 1993 bis 2002 mit jeweils 1\% stabile Quoten aufweisen - zu verzeichnen war, haben in den letzten Jahren eher differenziertere Entwicklungen in den verschiedenen Subgruppen stattgefunden [22]. Während der Aufwärtstrend bei den 13-jährigen Jungen und Mädchen sowie bei den 15-jährigen Mädchen deutlich abgebremst scheint (siehe Abb. 1), haben die 11- und die 15-jährigen Jungen bis 2001/2002 weiter zugelegt. Allerdings fällt dieser Zuwachs bei den 15-Jährigen relativ geringer aus als in der Vorperiode zwischen 1993/94 und 1997/98, und der konstante Zuwachs bei den 11-Jährigen von $1 \%$ über $2 \%$ auf $3 \%$ ist aufgrund der niedrigen Ausgangsraten und der niedrigen absoluten Fallzahlen eher mit Vorsicht zu interpretieren. $\mathrm{Zu}$ vermuten ist, dass die Stabilisierungstendenzen nicht zuletzt auf die deutliche Tabaksteuererhöhung von 2001/2002 zurückzuführen sind. Die aktuelle Drogenaffinitätsstudie der BZgA von 2004 geht davon aus, dass der Aufwärtstrend der neunziger Jahre inzwischen eindeutig gebrochen ist und führt dies ebenfalls wesentlich auf die Tabaksteuererhöhung zurück [23]. Ob sich ein solcher Trendbruch bzw. eine -umkehr gerade auch angesichts der erneuten Steuererhöhungen - auch in den HBSC-Daten niederschlagen wird, wird die nächste Befragungswelle von 2005/06 zeigen.

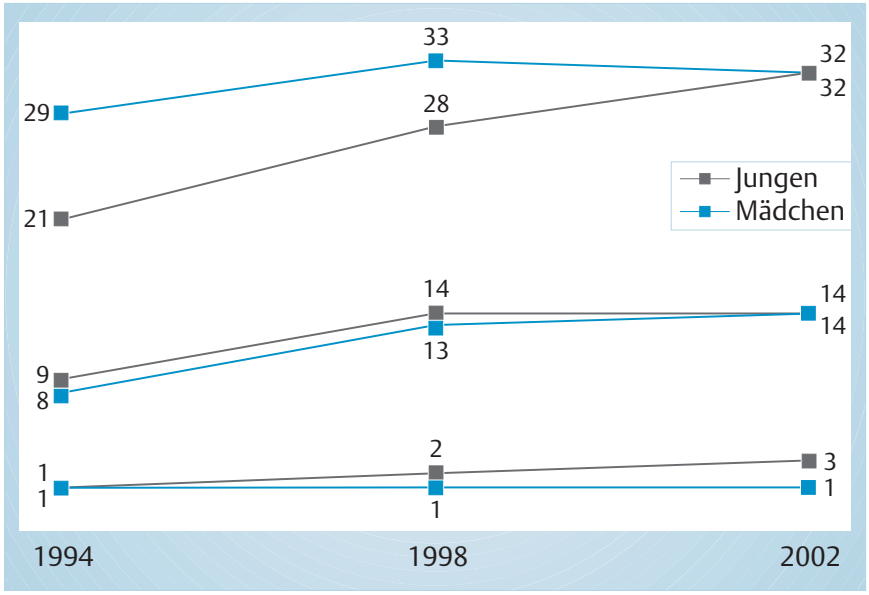

Abb. 1 Entwicklung des regelmäßigen Tabakkonsums: Vergleich der Quoten von 1994, 1998 und 2002 in NRW nach Alter und Geschlecht (Angaben in Prozent).

\section{Alkoholkonsum}

\section{Häufigkeit des Alkoholkonsums}

Wie beim Rauchen vollzieht sich der sozialisatorische Gewöhnungsprozess an die Alltagsdroge Alkohol graduell. Anders als beim Rauchen wird hier jedoch tatsächlich die große Mehrheit der Jugendlichen aktiv in den Konsumprozess integriert. Beträgt die Quote derjenigen, die aktuell keinen Alkohol trinken, bei den 11-Jährigen noch $79 \%$, sind es in Klasse 7 bei den 13-Jährigen nur noch 51,2\%. Danach scheint sich der Prozess zu beschleunigen und zwei Jahre später, bei den 15-Jährigen, sind es nur noch 19,1 \%, die sagen, sie hätten in der letzten Zeit keinen Alkohol ge- 
trunken $\left(\chi^{2=} 792,76 ; \mathrm{df}=2 ; \mathrm{p}<, 001\right)$. Der Prozentsatz der aktuell abstinenten Mädchen liegt insgesamt mit 53,5\% etwas über dem der abstinenten Jungen $(49,2 \%)(\mathrm{OR}=1,19 ; \mathrm{CI}=1,04-1,36)$.

Während ein Experimentieren mit Alkohol selbst bei jüngeren Jugendlichen nicht per se als problematisch betrachtet werden muss, sieht dies anders aus, wenn der Griff zum alkoholischen Getränk regelmäßig erfolgt, wobei Gebrauchshäufigkeiten ab einmal wöchentlich als regelmäßig eingestuft werden. Eine solche Regelmäßigkeit ist jedoch nur bei einer Minderheit (14,4\%) aller Jugendlichen zu beobachten. Dabei sind Jungen hier erwartungsgemäß stärker vertreten als Mädchen (siehe Tab.2) $(\mathrm{OR}=1,58 ; \mathrm{CI}=1,30-1,93)$, und mit dem Alter steigen die Quoten bei Mädchen wie bei Jungen deutlich an. Für die Gesamtgruppe erhöht sich der Anteil der regelmäßigen Konsumenten von 2,9\% bei den Fünftklässlern über 10\% in Klasse 7 auf 32,3\% bei den 15-Jährigen in Klasse $9\left(\chi^{2}=410,23 ; \mathrm{df}=2 ; \mathrm{p}<, 001\right)$.

Das am stärksten verbreitete alkoholische Getränk unter den 11bis 15-jährigen Jugendlichen aus NRW, die 2002 regelmäßig Alkohol trinken, ist nach wie vor das Bier. $11,4 \%$ geben an, in der letzten Zeit zumindest einmal wöchentlich Bier getrunken zu haben. An zweiter Stelle folgen mit 8,2\% alkoholische Mixgetränke, wozu vor allem die Alkopops gehören, Fertigmixturen von Süßgetränken und hochprozentigen Spirituosen, die in den letzten Jahren in Westeuropa bei Jugendlichen zunehmend an Beliebtheit gewonnen haben, aber auch die seit einigen Jahren immer beliebter werdenden Limonaden-/Cola-Biermischungen. Im Vergleich dazu ist regelmäßiger Wein- und Schnapskonsum deutlich seltener. Nur 2,6\% haben mindestens einmal wöchentlich Wein getrunken, bei Schnaps sind es, eigenen Angaben zu Folge, $4,1 \%$.

Altersspezifische Analysen zeigen, dass diese Rangfolge für fast alle Altersgruppen Gültigkeit hat. Nur bei den 11-Jährigen rangieren die alkoholischen Mixgetränke inzwischen vor dem Bier auf Platz 1. 2,3\% aller 11-Jährigen geben an, mindestens einmal im Monat Bier getrunken zu haben, bei den Mischgetränken sind es dagegen $4,1 \%$.

\section{Häufigkeit von Rauscherfahrungen}

Gut ein Viertel (28,3\%) der befragten Jugendlichen gibt an, schon einmal einen Rausch gehabt zu haben bzw. richtig betrunken gewesen zu sein, wobei auch hier die Frequenz primär vom Alter abhängt (siehe Tab. 2). Jungen und Mädchen unterscheiden sich weniger bezüglich der Rauscherfahrung an sich als bezüglich deren Häufigkeit, wobei die Unterschiede mit zunehmender Anzahl der Trunkenheitserfahrungen größer werden. So wird eine Häufigkeit von 10-mal und mehr nur von 2,8\% der Mädchen, aber von $6,1 \%$ der Jungen angegeben $(\mathrm{OR}=1,69 ; \mathrm{CI}=1,32-2,18)$.

\section{Innerdeutscher Vergleich der Konsumquoten}

Im Vergleich der Bundesländer hat Nordrhein-Westfalen mit $51,4 \%$ den höchsten Anteil an noch abstinenten Jugendlichen (Hessen: 47,1\%, Sachsen: 41,8\%, Berlin: 46,9\%), und auch wenn man regelmäßigen Konsum zum Kriterium macht, liegen die Jugendlichen aus Hessen (17,2\%) und Sachsen (16,8\%) mit ihren Quoten höher als die aus NRW, die mit $14,4 \%$ vertreten sind $(\mathrm{OR}=1,23 ; \mathrm{Cl}=1,03-1,49 / \mathrm{OR}=1,21 ; \mathrm{CI}=.97-1,51)$. Keine Unterschiede gibt es dagegen zu den Berliner Jugendlichen (12,8\%).

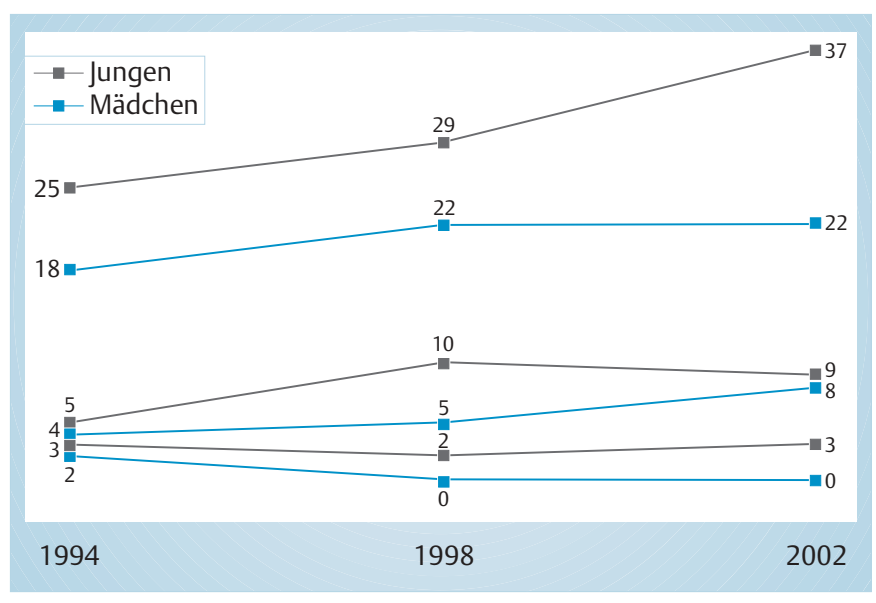

Abb. 2 Entwicklung des regelmäßigen Alkoholkonsums (alle Getränke zusammen): Vergleich der Quoten von 1994, 1998 und 2002 in NRW nach Alter und Geschlecht (Angaben in Prozent).

\section{Internationaler Vergleich der Konsumquoten}

Ein Blick auf den internationalen Kontext lässt allerdings, wie beim Rauchen auch, einen weniger günstigen Eindruck entstehen. Zwar schneiden die deutschen und speziell nordrhein-westfälischen Jugendlichen bei den 11-Jährigen noch recht gut ab, was den regelmäßigen Konsum von Alkohol angeht: mit Rang 33 liegen die Jungen im internationalen Vergleich ganz hinten, die Mädchen mit Rang 27 ebenfalls deutlich unter dem Durchschnitt aller 35 HBSC-Länder bzw. -Regionen [17, 21]. Allerdings scheinen sich in diesen geringen Raten vor allem relative Entwicklungsverzögerungen auszudrücken, die von den älteren Jahrgängen wieder eingeholt werden. Deutlich anders stellt sich das Bild nämlich bereits bei den 13-Jährigen dar, die den Durchschnitt mit Rang 17 bei den Jungen erreichen bzw. mit Rang 8 bei den Mädchen überschreiten. Bei den 15-Jährigen liegen dann Jungen (siebter Rang) als auch Mädchen (neunter Rang) über dem Durchschnitt aller Länder. Allerdings haben vor allem Mädchen aus anderen westeuropäischen Ländern wie England, Schottland und Wales, Dänemark und den Niederlanden zum Teil noch deutlich höhere Raten zu verzeichnen [17, 21].

\section{Entwicklung im Zeitverlauf}

Vergleicht man die Konsumquoten von 2001/2002 mit denen der HBSC-Studie von 1993/94 sowie 1997/98 zeichnet sich ein eindeutiger Anstieg ab [22]1. Eine differenzierte Betrachtung zeigt, dass diese Steigerung wesentlich auf veränderte Konsummuster bei den 15-jährigen Jungen zurückgeht (siehe Abb. 2). Waren es 1993/94 noch 25\%, die regelmäßig Alkohol tranken, ist dieser Prozentsatz im Jahr 2001/2002 um 12 Prozentpunkte auf 37\% angestiegen. Bei den Mädchen ist zwar auch ein Anstieg zu verzeichnen, allerdings fällt dieser mit $18 \%$ auf $22 \%$ weniger deutlich aus. Ähnlich zeigen sich im Zeitvergleich für die 13und 15-jährigen Jungen auch Anstiege in der Häufigkeit, mit der von zwei bis drei oder mehr Alkoholräuschen berichtet wird. Bei den 13-Jährigen stieg die Frequenz, mit der mehrfache Rausch-

\footnotetext{
1 Der für den zeitlichen Vergleich verwendete Alkoholindex basiert nur auf dem Konsum von Bier, Wein und Schnaps, da die alkoholischen Mixgetränke vor 2001 nicht in die Berechnung eingegangen sind. Hieraus ergeben sich auch Differenzen zu den in Tab. 2 angegebenen Quoten, die höher ausfallen als in Abb. $\mathbf{2}$.
} 
erfahrungen berichtet wurden, von 7\% im Jahr 1993/94 auf 13\% im Jahr 2001/2002 und von 34\% auf 44\% bei den 15-Jährigen. Bei den Mädchen ist die Steigerung dagegen vergleichsweise gering.

Da 1997/98 der Konsum von Alkopops noch nicht erhoben wurde, sind diese Getränke in den dargestellten Veränderungsquoten nicht enthalten. Allerdings ist davon auszugehen, dass sie zumindest teilweise über die Erfassung der Kategorien „Bier“ und „Schnaps“ indirekt mit eingegangen sind. Trotzdem liegt es nahe, anzunehmen, dass die Steigerungsraten de facto eher noch höher ausfallen dürften [siehe auch 23]. Die Alkoholspirituosen und Alkohollimonaden sind besonders auf eine junge Käuferschicht ausgerichtet und haben die Geschmackspräferenzen dieser Zielgruppe offensichtlich gut getroffen. Sie erleichtern gerade den jüngeren Jugendlichen den Übergang von den süßen Getränken der Kindheit zu den Erwachsenengetränken „Bier“, „Wein“ und „Schnaps“, die geschmacklich aufgrund ihres eher bitteren oder säuerlichen Charakters für viele anfänglich eher abschreckend wirken. Abzuwarten bleibt, ob die 2004 eingeführten Steuererhöhungen auf Alkopops diesen Konsumtrend abbremsen können.

\section{Cannabiskonsum}

\section{Häufigkeit des Cannabiskonsums}

Knapp ein Viertel der 15-Jährigen, nämlich 23,7\%, gibt an, bereits mindestens einmal Cannabis konsumiert zu haben. Diese Rate liegt zwar deutlich niedriger als die Prävalenzen für die legalen Drogen Tabak und Alkohol, bedeutet aber auch, dass die illegale Droge Cannabis durchaus Teil der jugendlichen Alltagskultur geworden ist. Dabei stellen mit 9,5\% allerdings diejenigen, die lediglich ein- oder zweimal probiert haben, die größte Teilgruppe, weitere 6,8\% haben bis zu 9-mal Cannabis genommen. Größere Häufigkeiten von 10- bis 39-mal bzw. von über 40-mal geben dagegen nur 3,2\% bzw. 4,3\% der Befragten an.

Etwas niedriger liegen die Quoten für die Einjahresprävalenz, die die aktuelle Konsumsituation widerspiegelt (siehe Tab.2). Hier sind es $17,7 \%$, die berichten, wenigstens einmal Cannabis genommen zu haben, bei weiteren $10,9 \%$ waren es bis zu 5-mal und nur $6,8 \%$ geben eine Häufigkeit über 5-mal an. Insgesamt scheint es sich bei den bis 15-Jährigen also eher um Probierkonsum zu handeln, der nicht notwendigerweise zu stabilen Verhaltensmustern führt. Hierfür spricht auch, dass immerhin fast ein Viertel (24,4\%) derjenigen, die schon einmal Cannabis genommen haben, angeben, im letzten Jahr nicht konsumiert zu haben.

Bei Jungen zeigt sich sowohl eine höhere Lebenszeitprävalenz $(29,1 \%)$ als bei Mädchen $(18,7 \%)(O R=1,78 ; C I=1,33-2,39)$ als auch eine höhere Einjahresprävalenz (Jungen: 22,4\% vs. Mädchen: $13,5 \%)(\mathrm{OR}=1,85 ; \mathrm{CI}=1,33-2,58)$. Noch augenfälliger werden die Unterschiede, wenn es um häufigeren Konsum geht. In der Gruppe derjenigen, die im vergangenen Jahr mehr als 5-mal Cannabis genommen haben, sind Jungen $(8,8 \%)$ erheblich stärker vertreten als Mädchen (2,7\%) (OR = 3,54; CI = 1,90-6,59).

\section{Innerdeutscher Vergleich der Konsumquoten}

Der Bundesländervergleich ergibt prägnantere Unterschiede für den Cannabiskonsum als für die Substanzen Tabak und Alkohol.
Jugendliche aus dem großstädtischen Berlin weisen dabei mit 33,5\% eine höhere Lebenszeitprävalenz auf ${ }^{2}$ als Schüler aus Hessen (24,9\%), NRW (23,7\%) und vor allem Sachsen (15,4\%). Ähnliche Differenzen gibt es auch bezüglich der Quoten für die häufiger, das heißt mehr als 5-mal im letzten Jahr, konsumierenden Jugendlichen. Dabei unterscheiden sich die Hessen und Sachsen mit 6,0\% und 3,7\% nicht signifikant von den NRW-Jugendlichen (5,6\%). Allerdings sind die Berliner Jugendlichen mit einem Anteil von $12,9 \%$ wiederum deutlich öfter als die Jugendlichen aus NRW in dieser Kategorie vertreten $(\mathrm{OR}=2,52 ; \mathrm{CI}=1,46-4,33)$.

\section{Internationaler Vergleich der Konsumquoten}

In Relation zu den Substanzen Alkohol und Tabak fällt der internationale Vergleich für die nordrhein-westfälischen Jugendlichen etwas günstiger aus. So liegen die NRW-Jugendlichen eher im Mittelfeld und entsprechen mit ihren Werten weitgehend dem Durchschnitt aller untersuchten Länder. Bei den Jungen nehmen sie sowohl bezüglich der Lebenszeitprävalenz als auch bezüglich der 12-Monatsprävalenz Rang 18, bei den Mädchen jeweils Rang 16 und Rang 18 ein. Andere westliche Industrieländer wie Kanada, USA, England, die Schweiz oder Frankreich weisen mit Raten von über 30\% bis zu 50\% deutlich höhere Konsumentenquoten auf [17, 21].

\section{Entwicklung des Cannabiskonsums}

Der Blick auf die Entwicklung des Cannabiskonsums im Vergleich zu 1997/98 3 zeigt allerdings, dass die Zahl der Konsumenten auch in Deutschland - zumindest in Subgruppen - wächst [siehe auch 24]. Während sich bei den Mädchen bezüglich des mindestens einmaligen Cannabiskonsums im Lauf der letzten zwölf Monate keine Veränderung ergeben hat - die Rate liegt hier konstant bei $15 \%$ - ist der entsprechende Anteil bei den männlichen Jugendlichen von $17 \%$ auf $22 \%$ angestiegen. Diese Angaben decken sich weitgehend mit denen der Drogenaffinitätsstudien von 2001 und 2004 [20, 23], die berichten, dass stabile Raten bei den 12- bis 25-jährigen Mädchen/jungen Frauen einem gestiegenen Konsum bei den Jungen/jungen Männern gegenüberstehen. Eine trotz der nach wie vor kontroversen politischen Debatte um das Gefahrenpotential der Substanz [25] größere Popularität von Cannabis legen auch die Befunde der BZgAStudie von 2004 zu Einstellungen gegenüber Cannabis nahe, die erkennen lassen, dass die Ablehnung gegenüber dieser speziellen illegalen Droge vor allem im Laufe der 90er-Jahre deutlich abgenommen hat [23].

\section{Fazit}

Unter den 11- bis 15-jährigen Jugendlichen in NRW sind Erfahrungen mit Tabak, Alkohol und selbst Cannabis ein „normales“ Phänomen: Etwa die Hälfte aller Befragten hat bereits mindestens einmal Zigaretten probiert, 50\% haben in der letzten Zeit Alkohol getrunken, 25\% bereits erste Erfahrungen mit Cannabis gemacht. Allerdings hat ein Großteil dieser Jugendlichen die Substanzen nur probiert oder konsumiert eher sporadisch. Der

\footnotetext{
2 Aufgrund der sehr geringen Stichprobengröße der Berliner Substichprobe sind die Befunde allerdings nur als bedingt stabil zu betrachten. 1993/94 wurde der Konsum illegaler Drogen nicht erfragt.
} 
Anteil der Jugendlichen, der bereits in diesem frühen Alter regelmäßig oder exzessiv psychoaktive Substanzen gebraucht, ist dagegen deutlich geringer. So sind circa $15 \%$ aller 11 - bis 15 -Jährigen zu den regelmäßigen Rauchern bzw. Alkoholkonsumenten zu rechnen, $4,4 \%$ berichten, mehr als 10 -mal betrunken gewesen zu sein, knapp 7\% haben im vergangenen Jahr mehr als 5-mal Cannabis konsumiert. Ein solch früher Einstieg in den habituellen oder intensiveren Konsum muss zumindest potenziell als gesundheitsriskant eingestuft werden. Besorgniserregend sind vor allem die relativ hohen Raten für den Tabakkonsum: Von den 15-Jährigen sind bereits fast ein Drittel regelmäßige Raucher, die damit international einen unrühmlichen Spitzenplatz beanspruchen können. Während noch abzuwarten bleibt, inwieweit die Tabaksteuererhöhungen der letzten Jahre in der nächsten Erhebung eventuell bereits erste Erfolge zeigen werden, demonstrieren die erfolgreichen Beispiele aus anderen Ländern, dass hier noch weitere Anstrengungen notwendig sind. Neben einer Verstärkung eher verhaltensorientierter schulischer Präventionsarbeit geht es vor allem um eine Intensivierung verhältnisorientierter Maßnahmen: von (weiteren) Tabaksteuererhöhungen, Abschaffung der Zigarettenautomaten zur vermehrten Schaffung rauchfreier Zonen, umfassenden Tabakwerbeverboten etc. [26].

Wie zu erwarten, ist das Konsumverhalten stark altersabhängig. Dabei fällt auf, dass der regelmäßige Konsum von Tabak und Alkohol bereits im Alter zwischen elf und 13 Jahren deutlich zunimmt - was eindeutig dafür spricht, mit suchtpräventiven Maßnahmen spätestens in der fünften Klasse, idealerweise aber bereits vorher zu beginnen. Andererseits kommen primärpräventive Anstrengungen zumindest für ein Drittel der 15-Jährigen eindeutig zu spät. Stattdessen muss es hier auch darum gehen, erste Rauchentwöhnungsangebote zu machen und präventive Maßnahmen speziell gegen exzessiven Alkohol- sowie gegen Cannabiskonsum anzubieten. Die Daten legen jedoch auch nahe, bei den Präventionsangeboten noch einmal nach Geschlecht zu differenzieren [27]. Zwar haben die Mädchen - und dies gilt besonders für das Rauchen - teilweise aufgeholt, das heißt, sie zeigen ähnliche Konsummuster wie Jungen. Allerdings - dies zeigen die bei den 11-Jährigen noch existierenden Geschlechtsunterschiede - setzt diese Entwicklung etwas später ein. Darüber hinaus sind extremere Konsumverhaltensweisen - dies wird vor allem bei den 15-Jährigen deutlich - nach wie vor eher eine männliche Domäne. Zudem zeigen auch die Verlaufsdaten, dass sich gerade bei den männlichen Jugendlichen in den letzten Jahren weitere Steigerungen im Konsum abzeichnen, so dass hier besonderer Interventionsbedarf zu bestehen scheint.

\section{Literatur}

${ }^{1}$ Engel U, Hurrelmann K. Was Jugendliche wagen. Weinheim: Juventa, 1993

${ }^{2}$ Hurrelmann K. Lebensphase Jugend. Weinheim: Juventa, 1999

${ }^{3}$ Silbereisen R, Kastner P. Jugend und Drogen. Entwicklung von Drogengebrauch - Drogengebrauch als Entwicklung. In: Oerter R (Hrsg) Lebensbewältigung im Jugendalter. Weinheim: VCH, 1985: 192-219

${ }^{4}$ Reese A, Silbereisen RK. Allgemeine versus spezifische Primärprävention von jugendlichem Risikoverhalten. In: Freund T, Lindner $\mathrm{W}$ (Hrsg). Prävention - Zur kritischen Bewertung von Präventionsansätzen in der Jugendarbeit. Opladen: Leske und Budrich, 2001: 139-162
5 diFranza JR, Rigotti NA, McNeill A et al. Initial symptoms of nicotine dependence in adolescents. Tobac Contr 2000; 9: 313-319

${ }^{6}$ Shedler J, Block J. Adolescent drug use and psychological health: a longitudinal inquiry. Am Psychol 1990; 45: 612 -630

${ }^{7}$ Roth M. Verbreitung und Korrelate des Konsums legaler und illegaler Drogen bei Jugendlichen. Zeitschrift für Gesundheitspsychologie 2002; 10: $23-35$

${ }^{8}$ Hawkins JD, Catalano RF, Miller JY. Risk and protective factors for alcohol and other drug problems in adolescence and early adulthood: implications for substance abuse prevention. Psych Bull 1992; 112: 64- 105

${ }^{9}$ Jessor R. New perspectives on adolescent risk behaviour. New York: Cambridge University Press, 1998

${ }^{10}$ Leppin A. Alkoholkonsum und Alkoholmissbrauch bei Jugendlichen. In: Leppin A, Hurrelmann K, Petermann $\mathrm{H}$ (Hrsg). Jugendliche und Alltagsdrogen. Neuwied: Luchterhand, 2000: 64-94

${ }^{11}$ Nordlohne E. Die Kosten jugendlicher Problembewältigung. Weinheim: Juventa, 1992

${ }^{12}$ Moffit TE. Adolescence-limited and life-course persistent antisocial behaviour: a developmental taxonomy. Psychol Rev 1993; 100 $674-701$

${ }^{13}$ Kaminer Y. Adolescent substance abuse: a comprehensive guide to theory and practice. New York: Plenum Press, 1994

${ }^{14}$ Ihle W, Esser G, Laucht M et al. Depressive Störungen und Verhaltensstörungen in Kindheit und Jugend. Prävalenz, Verlauf und Risikofaktoren. Bundesgesundheitsblatt Gesundheitsforschung und Gesundheitsschutz 2004; 47: $728-735$

${ }^{15}$ Pulkkinen L, Pitthauen P. A prospective study of the precursors of problem drinking in young adulthood. Journal of Studies on Alcohol 1994; $55: 578-587$

${ }^{16}$ Currie C, Samdal O, Boyce W et al (eds). Health behaviour in schoolaged children: a WHO cross-national study (HBSC), research protocol for the 2001/2002 survey. Edinburgh: Child and Adolescent Health Research Unit (CAHRU), 2001

${ }^{17}$ Currie C, Roberts C, Morgan A et al (eds). Young people's health in context - health behaviour in school-aged children. (HBSC) study: international report from the 2001/2002 survey. Kopenhagen: WHO-Europe, 2004

${ }^{18}$ Richter M. Anlage und Methode des Jugendgesundheitssurveys. In: Hurrelmann K, Klocke A, Melzer W et al (Hrsg). Jugendgesundheitssurvey - Internationale Vergleichsstudie im Auftrag der Weltgesundheitsorganisation WHO. Weinheim: Juventa, 2003: 9-18

${ }^{19}$ Richter M. Gesundheit und Gesundheitsverhalten im Jugendalter. Der Einfluss sozialer Ungleichheit. Wiesbaden: VS-Verlag, (im Druck)

${ }^{20}$ Bundeszentrale für gesundheitliche Aufklärung (BZgA). Die Drogenaffinität Jugendlicher in der Bundesrepublik Deutschland 2001. Eine Wiederholungsbefragung der Bundeszentrale für gesundheitliche Aufklärung. Köln: BZgA, 2001

${ }^{21}$ Richter M, Langness A, Hurrelmann K. Gesundheit und Gesundheitsverhalten von Schülerinnen und Schülern im internationalen Vergleich. Ergebnisse der internationalen WHO-Studie „Health Behaviour in School-aged Children (HBSC). Ergänzungsbericht zum International Report. Bielefeld: WHO Collaborating Centre, 2004

${ }^{22}$ Richter M, Settertobulte W. Gesundheits- und Freizeitverhalten von Jugendlichen. In: Hurrelmann K, Klocke A, Melzer W et al (Hrsg). Jugendgesundheitssurvey. Weinheim: Juventa, 2004: 99-158

23 Bundeszentrale für gesundheitliche Aufklärung (BZgA). Die Drogenaffinität Jugendlicher in der Bundesrepublik Deutschland 2004. Rauchen, Alkoholkonsum, Cannabis - Eine Wiederholungsbefragung der Bundeszentrale für gesundheitliche Aufklärung. Köln: BZgA, 2004

${ }^{24}$ Appel E, Hahn A. Verbreitung legalen und illegalen Drogenkonsums und die Bedeutung der Gleichaltrigen für die Drogeneinnahme bei Berliner Gymnasiasten. Zeitschrift für Gesundheitspsychologie 2001; 9: $13-25$

${ }^{25}$ Kleiber D, Soellner R. Cannabiskonsum. Entwicklungstendenzen, Konsummuster und Risiken. Weinheim: Juventa, 1998

${ }^{26}$ DKFZ. Gesundheit fördern - Tabakkonsum verringern. Handlungsempfehlungen für eine wirksame Tabakkontrollpolitik in Deutschland. Heidelberg: DKFZ, 2002

${ }^{27}$ Kolip P. Das gleiche ist nicht dasselbe. Zur Notwendigkeit geschlechtsspezifischer Suchtprävention im Jugendalter. Unterrichtswissenschaft 1997; 25: $150-160$ 